\title{
Precision of Biomarkers to Define Chronic Inflammation in CKD
}

\author{
Robert LaClair $^{a} \quad K^{2}$ alisha O'Neal ${ }^{a}$ Susan Ofner ${ }^{a}$ Marcelo J. Sosa ${ }^{b}$ \\ Carlos A. Labarrere ${ }^{b}$ Sharon M. Moe ${ }^{a, c}$ \\ ${ }^{\mathrm{a}}$ Indiana University School of Medicine, ${ }^{\mathrm{b}}$ Methodist Research Institute, and ${ }^{\mathrm{C}}$ Roudebush VAMC, \\ Indianapolis, Ind., USA
}

\section{Key Words}

Biomarkers, precision - Chronic inflammation · Chronic kidney disease $\cdot$ CKD stage 5D $\cdot$ Inflammatory biomarkers, intrapatient variance $\cdot$ Tunneled dialysis catheter

\begin{abstract}
Background/Aims: Several inflammatory biomarkers have been found to be associated with cardiovascular disease or all-cause mortality in dialysis patients, but their usefulness in clinical practice or as surrogate endpoints is not certain. The purpose of the present study was to determine the intrapatient variation of C-reactive protein, IL-6, fetuin-A and albumin in a population of dialysis patients. Methods: Apparently healthy dialysis patients with either a tunneled dialysis catheter or fistula had monthly assessments of these biomarkers for a total of four determinations, and the intraclass correlation coefficients were calculated as measures of intersubject variance. Results: Our results showed large within-subject variation relative to the total variation in the measurements (31-46\%). Having a tunneled catheter as opposed to a fistula was not significantly associated with mean levels, suggesting that chronic subclinical catheter infection does not explain the variation seen in the biomarkers. In contrast, there was a rapid change in these biomarkers with a clinically apparent acute infection. Conclusion: These results suggest that these biomarkers have limitations for use as surrogate endpoints in clinical trials due to wide fluctuations, even in apparently clinically healthy individuals.
\end{abstract}

Copyright $\odot 2008$ S. Karger AG, Basel

\section{KARGER}

๑ 2008 S. Karger AG, Basel

Fax +41613061234 E-Mail karger@karger.ch www.karger.com www.karger.com/ajn
Inflammation has been implicated in the etiology of coronary artery disease in the general population; biomarkers such as C-reactive protein (CRP) have been shown to predict cardiovascular events in both symptomatic and asymptomatic individuals [1]. Inflammatory markers have also been associated with carotid intimal thickness and coronary artery calcification $[2,3]$ in the general population. Importantly, single measurements of elevated levels of biomarkers such as CRP and interleukin-6 (IL-6), and lower levels of fetuin-A and albumin in patients with CKD stage 5D (on dialysis) are associated with increased mortality [4-7]. These data suggest that dialysis patients may have chronic inflammation, but the cause of inflammation has not been clearly delineated. Several possible etiologies have been implicated including, but not limited to, tunneled dialysis catheters, oxidative stress, non-pure water, and periodontal disease [810].

Given the data demonstrating a strong association between biomarkers of inflammation and mortality in CKD stage 5D, the next logical step is to intervene with a therapy to reduce inflammation in order to improve cardiovascular disease and/or all-cause mortality. However, such a study requires a large population. A preferred intermediate step is an interventional trial with an agent to reduce inflammation using a surrogate biomarker as an endpoint. The Food and Drug Administration defines a biomarker as 'a characteristic that is objectively measured and evaluated as an indicator of normal biologic or pathogenic processes or pharmacological responses to a thera-

Sharon M. Moe, MD

Indiana University School of Medicine

1001 W. 10th Street, OPW 526

Indianapolis, IN 46202 (USA)

Tel. +1 317278 2868, Fax +1 317278 2860, E-Mail smoe@iupui.edu 
peutic intervention' [11]. Biomarkers are often used in early drug development to establish proof of concept as a surrogate endpoint in clinical trials. However, the use of a biomarker as a surrogate endpoint requires demonstration that it correlates with the clinical endpoint (accuracy) and that it is reproducibly measured (precision) [11]. An estimate of the within-subject variance of a biomarker could facilitate the design of a clinical study.

The purpose of the present study was to determine the intrapatient variance of inflammatory biomarkers in CKD stage 5D patients and to determine if the presence of a tunneled dialysis catheter (compared to fistula) explained some of the variation levels of these biomarkers. A second aim was to observe changes in these markers following a clinically identifiable acute event.

\section{Methods}

The primary aim of the study was to determine the intrapatient variance of biomarkers of inflammation in patients on dialysis and whether tunneled dialysis catheters (TC) explained the variation. To examine this, we measured CRP, IL-6, fetuin-A, and albumin in patients who were clinically without acute medical complaints at baseline and monthly thereafter for 3 months. Monthly measurements were chosen so as to coincide with other routine blood draws. Patients were interviewed for clinical symptoms weekly by the nurse practitioner and monthly by the investigators to assess acute clinical events. The second aim of the study was to determine the timing and magnitude of change in these biomarkers in subjects who developed a clinical event such as infection or other significant morbidity. For the latter, we measured weekly levels of these biomarkers beginning at the onset of illness and continuing for 2 months.

The inclusion criterion was an AVF or TC as an access (i.e. no grafts) in use for at least 2 months. This inclusion criterion was to clearly distinguish those patients with and without foreign objects that may serve as a nidus for infection. Exclusion criteria included inability to give informed consent, significant liver disease (cirrhosis or liver function tests three times normal), a chronic infectious process (HIV, osteomyelitis) or malignancy; life expectancy $<6$ months; on immunosuppressants. Subjects were also excluded if they had a significant infection in the previous 2 months defined as requiring hospitalization, positive blood cultures, positive chest $\mathrm{x}$-ray for pneumonia, 50\% increase in total WBC count, myocardial infarction, stroke or other event requiring hospitalization including access declotting procedures. The study was approved by the IUPUI/Clarian Institutional Review Board.

All serum was collected before dialysis after a short (2-day) interval at the beginning of each calendar month for 3 months, immediately aliquoted, and frozen at $-80^{\circ} \mathrm{C}$ until analyzed at the end of the study by batch analyses. Fetuin-A levels were measured by ELISA (Epitope Diagnostics, Inc.). IL- 6 was measured by ELISA (R\&D Laboratories), and high sensitivity (CRP) was analyzed by ELISA (Alpha Diagnostics International). Serum albumin lev- els were analyzed by bromocresol purple methodology (ALBm, Synchron ${ }^{\circledR}$, Beckman Coulter). The coefficient of variation (CV) for the kits in our laboratory were 2.2\% (albumin), 7.3\% (IL-6 and CRP) and 9.6\% (fetuin-A).

\section{Statistical Methods}

Intraclass correlation coefficients (ICC) were calculated as measures of intersubject variance. The ICC is a measure of the proportion of variation resulting from measurement of different subjects. Since the total variation consists of variation coming from repeated measurements of the same subject and from measurements of different subjects, one minus the ICC (1 - ICC) gives us the proportion of variation that comes from repeatedly measuring the same subject. In other words, 1 - ICC expresses the magnitude of within-subject variance relative to total variance.

Both CRP and IL- 6 showed deviation from normality, so each of these was log transformed for modeling purposes. Baseline characteristics, medication usage and laboratory values albumin, fetuin-A, $\log (\mathrm{CRP})$, and $\log (\mathrm{IL}-6)$ levels were compared between groups by use of an analysis of variance (ANOVA) model or $\chi^{2}$. Correlations of measurements at each visit were estimated by Spearman's rank. Longitudinal measurements of albumin, fetuin-A, $\ln (\mathrm{CRP})$ and $\ln (\mathrm{IL}-6)$ were modeled using a repeated measures ANOVA with terms for group (AVF vs. TC), visit and the group by visit interaction. For fetuin-A there was one outlier, subject 20 at visit 3 . The outlier was removed for the analysis of fetuinA and the model refit. Other patient characteristics (age, gender, race, duration of dialysis, diabetes, being a smoker) were tested for association with longitudinal measures of the biomarkers by use of a linear model which included a term for the patient characteristic and visit. A random patient effect was also included to incorporate into the model the correlation of measurements from the same patient.

\section{Results}

92 subjects were approached from three dialysis units affiliated with Indiana University for the research study; 69 consented. 12 consented subjects were not included in the primary aim (monthly levels) of the study because of a clinical event during the study and thus they were no longer clinically stable: 4 of these subjects had an infection (4 TC, 0 fistula), 3 underwent a kidney transplant, 3 changed to daily dialysis, 1 had a change in dialysis access, and 1 patient had a cerebral vascular accident. $\mathrm{Pa}$ tient demographic and baseline laboratory values are shown in table 1 . The only significant differences between fistula and TC patients were dialysis vintage and diabetes. There were significant, but not strong correlations between inflammatory markers at baseline. FetuinA correlated with IL- $6(\mathrm{r}=-0.34, \mathrm{p}=0.01)$, IL-6 correlated with CRP $(\mathrm{r}=0.39, \mathrm{p}=0.002)$, albumin correlated with IL-6 $(\mathrm{r}=-0.45, \mathrm{p}<0.001)$ but there were no correlations between CRP and albumin or CRP and fetuin-A. 
Table 1. Patient characteristics at baseline

\begin{tabular}{|c|c|c|}
\hline & $\begin{array}{l}\text { AVF } \\
(\mathrm{n}=32)\end{array}$ & $\begin{array}{l}\text { TC } \\
(\mathrm{n}=26)\end{array}$ \\
\hline Age, years & $50 \pm 15$ & $55 \pm 14$ \\
\hline Male/female & $21 / 11$ & $11 / 15^{*}$ \\
\hline Race (African-American/Caucasian) & $24 / 8$ & $21 / 5$ \\
\hline Dialysis vintage, months & $84.2 \pm 92.3$ & $42.5 \pm 53.5^{* *}$ \\
\hline Cause of ESRD: GN/HTN/DM/other & $9 / 13 / 7 / 3$ & $3 / 8 / 13 / 2$ \\
\hline Diabetes, yes/no & $9 / 23$ & $15 / 11^{* *}$ \\
\hline Current smoker, yes/no & $10 / 22$ & $6 / 20$ \\
\hline $\begin{array}{l}\text { Hospitalization in last } 12 \text { months } \\
\text { yes/no }\end{array}$ & $12 / 20$ & $12 / 14$ \\
\hline Previous cardiac event, yes/no & $8 / 24$ & $6 / 20$ \\
\hline Previous stroke or TIA, yes/no & $4 / 28$ & $6 / 20$ \\
\hline PVD, yes/no & $4 / 28$ & $3 / 23$ \\
\hline Hepatitis $C$, yes/no & $7 / 25$ & $2 / 24$ \\
\hline Statin, yes/no & $17 / 15$ & $9 / 17$ \\
\hline Average weekly darbepoetin dose, $\mu \mathrm{g}$ & $57 \pm 52$ & $60 \pm 56$ \\
\hline Vitamin D, yes/no & $20 / 12$ & $13 / 13$ \\
\hline Hct, \% & $37.4 \pm 4.3$ & $36.4 \pm 6.6$ \\
\hline $\mathrm{WBC}$, cells $/ \mathrm{mm}^{3}$ & $7.2 \pm 2.3$ & $7.7 \pm 2.6$ \\
\hline Ferritin, ng/ml & $677 \pm 265$ & $610 \pm 399$ \\
\hline PTH, pg/ml & $563 \pm 508$ & $554 \pm 683$ \\
\hline Calcium, mg/dl & $8.9 \pm 0.8$ & $9.2 \pm 0.6$ \\
\hline Phosphorus, mg/dl & $5.6 \pm 1.5$ & $5.3 \pm 1.6$ \\
\hline Albumin, $\mathrm{g} / \mathrm{dl}$ & $3.8 \pm 0.6$ & $3.6 \pm 0.7$ \\
\hline Fetuin-A, $\mu \mathrm{g} / \mathrm{ml}$ & $\begin{array}{l}335 \pm 92 \\
(172-548)\end{array}$ & $\begin{array}{l}378 \pm 99 \\
(142-562)\end{array}$ \\
\hline High-sensitivity CRP, ng/ml & $\begin{array}{r}12.32 \pm 15.92 \\
(0.6-65.5)\end{array}$ & $\begin{array}{r}21.94 \pm 30.4 \\
(0.7-91.1)\end{array}$ \\
\hline $\ln (\mathrm{CRP})$ & $1.80 \pm 1.25$ & $2.18 \pm 1.40$ \\
\hline IL-6, pg/ml & $\begin{array}{l}14.9 \pm 19.1 \\
(1.8-92.9)\end{array}$ & $\begin{array}{l}8.6 \pm 8.0 \\
(1.1-29.5)\end{array}$ \\
\hline $\ln (\mathrm{IL}-6)$ & $2.17 \pm 0.99$ & $1.78 \pm 0.87$ \\
\hline
\end{tabular}

Data presented as mean \pm SD and ranges for those values that were subsequently log transformed.

$\mathrm{AVF}=$ Arteriovenous fistula; $\mathrm{TC}=$ tunneled dialysis catheter. Cause of ESRD: GN = glomerulonephritis, HTN = hypertension, $\mathrm{DM}=$ diabetes.

${ }^{*} \mathrm{p}=0.08 ;{ }^{* *} \mathrm{p}<0.05$.

Table 2. Variation of inflammatory biomarkers

\begin{tabular}{lccccc}
\hline & $\begin{array}{l}\text { Between- } \\
\text { subject } \\
\text { variation }\end{array}$ & $\begin{array}{l}\text { Within- } \\
\text { subject } \\
\text { variation }\end{array}$ & $\begin{array}{l}\text { Within- } \\
\text { subject } \\
\text { SD }\end{array}$ & ICC & 1- ICC \\
\hline Fetuin-A & 7,759 & 6,722 & 82 & 0.54 & 0.46 \\
CRP & 376 & 1,224 & 35 & 0.24 & 0.76 \\
$\ln ($ CRP) & 1.27 & 0.57 & 0.75 & 0.69 & 0.31 \\
IL-6 & 108 & 326 & 18 & 0.25 & 0.75 \\
$\ln ($ IL-6) & 0.69 & 0.33 & 0.57 & 0.68 & 0.32 \\
Albumin & 0.11 & 0.20 & 0.45 & 0.36 & 0.64 \\
\hline
\end{tabular}

CRP = C-reactive protein; IL-6 = interleukin- 6 ; ICC = intraclass correlation coefficients.
When patients were examined longitudinally, the inflammatory markers changed frequently despite no clinically apparent illness. There was large month-to-month fluctuation for all biomarkers. To determine if some of this variation seen in clinically stable patients was explained by the presence of TC, we compared these biomarkers at baseline between patients with an AVF to those with a TC, and found no difference (table 1). We then analyzed all monthly measurements and found a significant difference between mean fetuin-A for patients with AVF as compared to those with TC at visit 3 only, but this was due to an outlier value from a single patient. Once this patient's value was removed, the difference became non-significant. There were no significant differences in $\ln (\mathrm{CRP}), \ln (\mathrm{IL}-6)$, or albumin between patients with AVF and those with TC at any visit.

The 1 - ICC calculation (table 2) demonstrated that $46 \%$ of total variation in fetuin-A levels comes from within-patient variance or each patient's fluctuations in monthly levels. Corresponding 1 - ICC was $31 \%$ for $\ln (\mathrm{CRP}), 32 \%$ for $\ln$ (IL-6), and $64 \%$ for albumin. $30 \%$ of subjects changed from one tertile of $\ln (\mathrm{CRP})$ to another during the 3-month study. Other patient characteristics were tested for association with biomarker levels. Smoking was the only patient characteristic that was significantly associated with any of the biomarkers. It was negatively associated with fetuin-A levels and positively associated with $\ln$ (IL-6).

Despite the large intrapatient variance of these biomarkers seen in these clinically stable patients, there was a predictable response to acute inflammation. Four subjects with infection during the study were followed weekly - all with TC. All of these patients had a sudden rise in CRP and IL-6, and drop in fetuin-A within 1 week of onset of the infection, and a return to baseline values within 3 weeks following the onset of the clinical symptoms of infection. The most serious infection was endocarditis, and the changes in inflammatory biomarkers for this subject are shown in figure 1 . No data for these patients were included in Aim 1 of the study (monthly) as Aim 1 sought to determine variation in clinically stable patients.

\section{Discussion}

Dialysis patients have a large burden of cardiovascular disease, with both traditional Framingham and many 'non-traditional' risk factors identified. One such nontraditional risk factor is inflammation. A syndrome of 


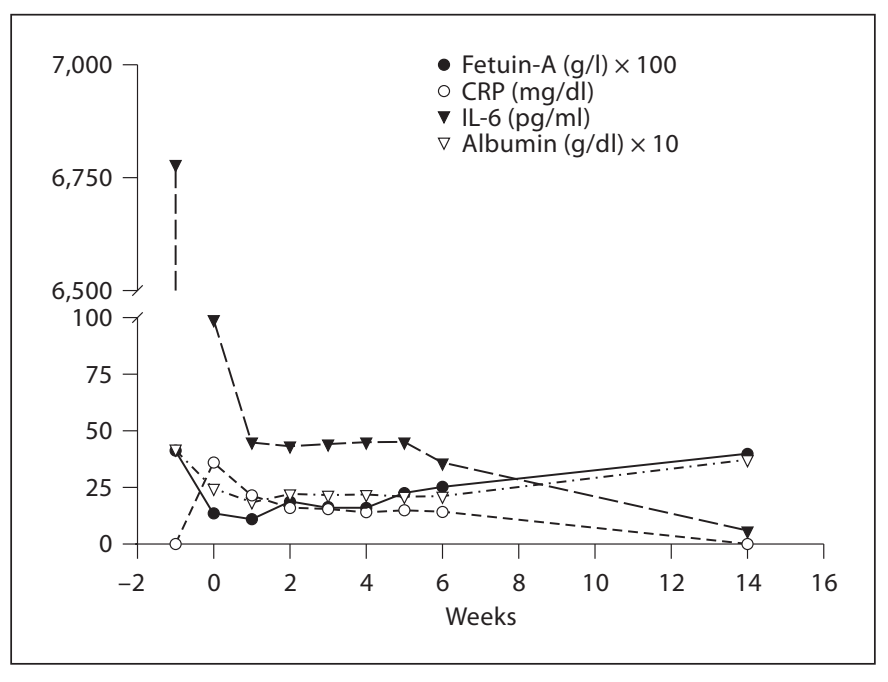

Fig. 1. Change in inflammatory markers in patient with endocarditis: a patient was diagnosed with acute infectious endocarditis 7 days after baseline (week 0 ) laboratory values. The patient had weekly inflammatory biomarkers measured to assess the change with antibiotic treatment and mitral valve replacement. To show the trends for all biomarkers on the same graph, the albumin levels were multiplied by 10 , and the fetuin-A was multiplied by 100.

malnutrition, inflammation and atherosclerosis has been identified in dialysis patients and implicated in the pathogenesis of cachexia and cardiovascular disease [9]. Pathologically, inflammation is a known contributor to intimal atherosclerotic disease in which macrophages and other inflammatory cells are thought to be critical in the pathogenesis of plaques [12]. Inflammation has been shown to lead to reduced fetuin-A levels in serum, likely making patients more vulnerable to extraskeletal calcification when this serum calcium-binding protein is decreased [13]. The advent of the high-sensitivity CRP has led to a plethora of studies in the general population and in dialysis patients - nearly all showing a strong association of elevated levels with cardiovascular and all-cause mortality. However, inflammation is an end event of numerous inciting factors and mediators, and many of the biomarkers utilized to define or diagnose inflammation are part of the acute phase response, which is a non-specific diffuse response initiated by multiple factors [14].

In the present study, we documented that these biomarkers change with acute events such as infection and stroke, with recovery as the event recovers. Thus the accuracy of the biomarkers for acute inflammation is not questioned. However, we found large variances in patients who were clinically stable, and this variance was not explained by having a TC as opposed to an AVF. TC have been hypothesized to contribute to intermittent inflammation and elevated biomarkers of inflammation [8]. In the present study, patients with TC were more likely to get infected, but in patients without clinically apparent infections, the presence of TC did not explain the variance. Between 30 and $46 \%$ of the total variation was due to within-patient variation despite assay performance (CV) that was within manufacturer recommendations.

Similar estimates of variance have been previously reported in a smaller study in dialysis patients. A study of 29 patients, excluding subjects with temporary catheters, measured levels weekly for 16 months and found intrasubject variance even higher, with 71.3 and $86.7 \%$ of total variation for CRP and IL-6, respectively [15]. An equally poor variance was found for serum amyloid A protein, another inflammatory biomarker [15]. In contrast, estimates of variation in the general population are much lower. In one study, the intraindividual variation for $\ln (\mathrm{CRP})$ for healthy adults was $14 \%$ compared to $9 \%$ for those with angina [16]. Thus, this variance is substantially higher in dialysis patients than in the healthy adults, even in those with native fistulas. The reason for the variation could not be assessed from this study as we did not perform random blood cultures, dental examinations, tagged white cell scans or other searches for clinically hidden causes of inflammation.

Unfortunately, the lack of stability of these biomarkers makes practical use problematic. Clinical use of the biomarkers to identify patients at risk or who have smoldering infections is not likely valid given that, for example, over one-third of subjects changed from one tertile of CRP to another in the course of just 3 months. This is consistent with the study of van Tellingen et al. [17], who found patients frequently changed tertiles of CRP and concluded that clinical judgment was as predictive of infection as was a rise in CRP levels. The intrapatient variation will need to be taken into account if any of these biomarkers are used as a surrogate endpoint in research studies that employ specific interventions aimed at reducing inflammation. Certainly, sample size calculations for studies using these biomarkers in dialysis patients should take into account this variation and study designs should include repeated measurements to be certain that the observed effect is stable over time. Our data support the need to validate that a change in these biomarkers prospectively predicts a change in clinical endpoints prior to their use as a valid surrogate endpoint. Lastly, authors should report their own laboratories' CV when using these assays. Nearly all samples analyzed in the pres- 
ent study required dilution of the sample to remain within the manufacturer's standard curve, which may create increased assay error.

There are several limitations to our study. First, this is a relatively small sample size although the largest reported to date. We only followed patients for 4 months, and perhaps longer follow-up, or more frequent follow-up would have altered our findings, although Tsirpanlis et al. [15], measuring weekly values found similar variability. We also only measured four potential inflammatory biomarkers, all of which have limited specificity for inflammation. Lastly, there may have been some selection bias in those patients enrolled. Our patients were predominately African-American, had a high percentage of glomerulonephritis and were younger than the average US dialysis population. However, these apparently clinically stable patients may be enrolled in clinical trials and thus the results provide important information on the limitations of using these biomarkers in clinical trials.

In summary, there is considerable intrapatient variation in biomarkers CRP, IL-6, fetuin-A and albumin.
Whether this phenomenon is a problem with the assays, a problem with our patients or both is a question that remains to be answered. Given problems with circulating fragments in other peptides such as PTH [18], interference in assay measurements by inactive fragment or other uremic toxins is a possibility. However, a more likely explanation for the high and fluctuating levels in dialysis patients is that these biomarkers can be elevated by a number of processes, and thus may simply reflect the multiple co-morbid illnesses present in our patients. Unfortunately, this means further studies are needed to refine the precision of these biomarkers before they can be considered true surrogate markers for cardiovascular disease in dialysis patients. Until that time, clinical studies of anti-inflammatory measures should evaluate clinically meaningful endpoints to prove efficacy.

\section{Acknowledgment}

This study was supported by the National Kidney Foundation of Indiana and NIH, K24 DK 002775.

\section{References}

1 Ridker PM: Clinical application of C-reactive protein for cardiovascular disease detection and prevention. Circulation 2003;107: 363-369.

-2 Stenvinkel P, Heimburger O, Paultre F, Diczfalusy U, et al: Strong association between malnutrition, inflammation, and atherosclerosis in chronic renal failure. Kidney Int 1999;55:1899-1911.

- 3 Moe SM, Chen NX: Inflammation and vascular calcification. Blood Purif 2005;23:6471.

-4 Lowrie EG, Lew NL: Death risk in hemodialysis patients: the predictive value of commonly measured variables and an evaluation of death rate differences between facilities. Am J Kidney Dis 1990;15:458-482.

5 Yeun JY, Levine RA, Mantadilok V, Kaysen GA: C-reactive protein predicts all-cause and cardiovascular mortality in hemodialysis patients. Am J Kidney Dis 2000;35:469476.

-6 Stenvinkel P, Barany P, Heimburger O, Pecoits-Filho R, et al: Mortality, malnutrition, and atherosclerosis in ESRD: What is the role of interleukin-6? Kidney Int Suppl 2002: 103-108.

7 Ketteler M, Bongartz P, Westenfeld R, Wildberger JE, et al: Association of low fetuin-A concentrations in serum with cardiovascular mortality in patients on dialysis: a crosssectional study. Lancet 2003;361:827-833.
8 Himmelfarb J, Stenvinkel P, Ikizler TA, Hakim RM: The elephant in uremia: oxidant stress as a unifying concept of cardiovascular disease in uremia. Kidney Int 2002;62: 1524-1538.

9 Stenvinkel P: Inflammation in end-stage renal failure: could it be treated? Nephrol Dial Transplant 2002;17(suppl 8):33-40.

-10 Franek E, Blaschyk R, Kolonko A, MazurPsonka L, et al: Chronic periodontitis in hemodialysis patients with chronic kidney disease is associated with elevated serum C-reactive protein concentration and greater intima-media thickness of the carotid artery. J Nephrol 2006;19:346-351.

11 Biomarkers Definitions Working Group: Biomarkers and surrogate endpoints: preferred definitions and conceptual framework. Clin Pharmacol Ther 2001;69:89-95.

12 Ridker PM, Hennekens CH, Buring JE, Rifai $\mathrm{N}$ : C-reactive protein and other markers of inflammation in the prediction of cardiovascular disease in women. $\mathrm{N}$ Engl J Med 2000;342:836-843.
13 Ketteler M, Wanner C, Metzger T, Bongartz $\mathrm{P}$, et al: Deficiencies of calcium-regulatory proteins in dialysis patients: a novel concept of cardiovascular calcification in uremia. Kidney Int Suppl 2003:S84-S87.

14 Pepys MB, Hirschfield GM: C-reactive protein: a critical update. J Clin Invest 2003;111: 1805-1812.

15 Tsirpanlis G, Bagos P, Ioannou D, Bleta A, et al: The variability and accurate assessment of microinflammation in haemodialysis patients. Nephrol Dial Transplant 2004;19: 150-157.

16 De Maat MP, de Bart AC, Hennis BC, Meijer $\mathrm{P}$, et al: Interindividual and intraindividual variability in plasma fibrinogen, TPA antigen, PAI activity, and CRP in healthy, young volunteers and patients with angina pectoris. Arterioscler Thromb Vasc Biol 1996;16: $1156-1162$.

17 Van Tellingen A, Grooteman MP, Schoorl M, Bartels PC, et al: Intercurrent clinical events are predictive of plasma C-reactive protein levels in hemodialysis patients. Kidney Int 2002;62:632-638.

- 18 Souberbielle JC, Boutten A, Carlier MC, Chevenne D, et al: Inter-method variability in PTH measurement: implication for the care of CKD patients. Kidney Int 2006; 70 : $345-350$ 